\title{
A Review on BCI Emotions Classification for EEG Signals Using Deep Learning
}

\author{
Puja A. Chavan ${ }^{\mathrm{a}, 1}$ and Dr. Sharmishta Desai ${ }^{\mathrm{b}}$ \\ ${ }^{a}$ School of Computer Engineering \& Technology, MIT World Peace University, \\ Pune, Maharashtra, India \\ ${ }^{b}$ Associate Professor, School of CET, MIT World Peace University
}

\begin{abstract}
Emotion awareness is one of the most important subjects in the field of affective computing. Using nonverbal behavioral methods such as recognition of facial expression, verbal behavioral method, recognition of speech emotion, or physiological signals-based methods such as recognition of emotions based on electroencephalogram (EEG) can predict human emotion. However, it is notable that data obtained from either nonverbal or verbal behaviors are indirect emotional signals suggesting brain activity. Unlike the nonverbal or verbal actions, EEG signals are reported directly from the human brain cortex and thus may be more effective in representing the inner emotional states of the brain. Consequently, when used to measure human emotion, the use of EEG data can be more accurate than data on behavior. For this reason, the identification of human emotion from EEG signals has become a very important research subject in current emotional braincomputer interfaces (BCIs) aimed at inferring human emotional states based on the EEG signals recorded. In this paper, a hybrid deep learning approach has proposed using CNN and a long short-term memory (LSTM) algorithm is investigated for the purpose of automatic classification of epileptic disease from EEG signals. The signals have been processed by CNN for feature extraction from runtime environment while LSTM has used for classification of entire data. Finally, system demonstrates each EEG data file as normal or epileptic disease. In this research to describes a state of art for effective epileptic disease detection prediction and classification using hybrid deep learning algorithms. This research demonstrates a collaboration of $\mathrm{CNN}$ and LSTM for entire classification of EEG signals in numerous existing systems.
\end{abstract}

Keywords. Deep learning, machine learning, DCNN, signal processing, social data analytics

\section{Introduction}

Nowadays the subfield of Brain Computer Interaction (BCI) encompasses everything we do with our Human Computer Interaction (HCI). It provides the ability to connect to electronic devices such as a computer and a cell phone with the human brain. BCI has played a significant role in helping disabled people. An EEG-based BCI framework involves a user who interacts with the hardware that employs other technologies as well. In the previous expandable BCI, several processing steps were taken to find the reason for brain signals and transform them into something that the user wanted to do. BCI techniques collect signals from the brain tissue, learn about these signals, and use this information to identify the subject's intentions.

${ }^{1}$ Puja A. Chavan, School of Computer Engineering \& Technology, MIT World Peace University, Pune, Email:apujachavan02@gmail.com. 
Similar to the imaging studies, electrodes may be used for non-medical purposes such as for sports, educating, tracking, and for entertainment purposes as well. Feelings are extremely important in human cognition, especially in rational decision-making and interpretation, in addition to helping people communicate and help them gain understanding. Recent affects-related computing appears to have caught up to HCI by linking emotions and systems with HCI, with this as well as other fields of computing Measuring emotional states using human-computer interactions refers to ascertains consumer engagement with the technology People may have an emotional awareness of other than a reaction to stimuli in their bodies, particularly for their emotional memories. psychology, neuroscience, and computer science continue to collaborate to improve analysis of emotion recognition [link to brain and thought processing working together] Curious to see how the algorithms of modern artificial intelligence could be applied to any form of modern society, modern militaries, manufacturing, and academic have always demonstrated and fantasy on computer screens. Alternative strategies include those that use emotional behavioral patterns such as well as well as facial and voice cues, including movement and tone of voice, but there are also the ones that employ additional factors, though less definite, clues like gut feelings and vague feelings as identifiers. Noninvasive sensors may typically record biological activity as well as well as electrochemical and/electrical signals. These models include conductivity, electrocardiogram, and electrocardiogram measurements.

\subsection{Overview of Deep learning:}

It is the sub-branch of machine learning that makes the machines smart enough so the machines are capable to learn from past experience and understand the real-world concepts. When a computer gathers more real-world intelligence, it becomes better at making decisions on what to do base on new experiences [4].

In Deep Learning, "deep" indicates the number of hidden layers in the Neural Networks. Deep Learning models are trained by using any large set of labeled data. Deep learning techniques are used for image sentiment analysis and providing optimum results. With increasing investment in deep learning, a lot of such approaches such as Convolutional Neural Network, Deep Belief Network, Deep Belief Network, and Deep Learning play an important roles in providing algorithms that utilize various features including word embedding, word embedding, word co-oriented belief, and connectionist analysis, to deliver optimal results, state of the art results It is also expressed as image and text here: When people have differing emotional conflicts, undesirable, or incommoded feelings, the main issue we see is in our studies is that these feelings are expressed in both image and text [5].

\section{Review of Literature}

A wide range of signal processing and machine learning experiments have been done on seizure recognition, many of which have included studies of the convolving features. the authors isolated the frequencies in [1] using the wavelet transform (WT), which provides an understanding of the frequency content of the signal, and its standard deviation, and the Lyapun's characteristics, as two sets, and then measured the signal's three main distinguishing features: variance and coherence. Depending on the method, several different methods have been found to be more suitable for seizures: 
wavelet analysis and the wavelet-based network learning system. in the article titled "Classifying Learning with Fourier Expansions, a Lifts, in [3], features were extracted with the FFT, and classification trees were used to analyse these features PCA review found that these two methods to be applicable to various models: Principal Component Analysis (PCA) and Genetic Algorithm (GA) were usable for all models of different types, from linear to non-linear. Computation of the initial principal variable using a non-linear algorithm came up with better results than a genetic algorithm. according to the paper, which showed a comparison of wavelet-based techniques for seizure detection, a 2004 saw in [4]. Expanding Spectral Epileptosis and Detection of Epileptiform Pulses and Training and Testing Sets" (also known as "expanding spectral epilepsy extract and test feature set extraction"), authors listed training and testing sets for recognizing and classifying seizure segments by extracting additional spectral features." Classification SVMs are helpful for purposes of identifying strong support vectors, for instance.

Using a strategy of the empirical mode decomposition of signals from the EEG to do the study It was used to pull out the real-valued components in the same way as the Hilbert-Huang transform (HHT). These functional capabilities were applied as features for the differentiation of EEG signals. using distinct brain regions, as well as groups of ECG cycles, [5] as opposed to individuals, according to the study [6] using distinctive regions of the brain, expanded the research in [8] used the non-non-linear dynamics of the Lyapunov exponent, and largest Lyapunov wavelet-based method to measure the EEG signals, both calculated from correlation coefficients, for study. This model used logistic trees to derive optimal seizure detection algorithms that draw on statistical features and incorporated a statistical detection technique that utilises the logistic feature space. Some statistical features were used as input to a logistic model (LMT) for the epileptic seizure recognition. This algorithm was used to validate the accuracy of the presented approach using an EEG dataset. Some of the papers previously discussed illustrate various classification objectives and feature extraction strategies. These models have been created for the systems they are considered to be essential features of the systems. Since the researchers in the paper [9] employed the discrete wavelet transform (DWT), the brain wave signals were subdivided into frequency bands and thus made into a collection of discrete statistics in [9] were obtained In order to reduce the amount of data, the PCA, independent components analysis (ICA) and linear discriminant analysis (LDA) were utilised. Once the extracted features had been added, SVM was used to distinguish non-epileptic seizures, the model got better at identifying epileptic seizures [10].

Instead of drawing an arbitrary pattern, interictal EEG data points were used in [in 11] to form a data Figure [a universal universe Figure] in [as a random.]. using feature extraction techniques and an implementation of Universum SVM, [Feature Vector Machine (SVM) Classifier], they were able to Structure out of important features in [12] from the EEG data was developed and made apparent, using NCA (neighbourhood component analysis). Also, they tried out AdaBoost (adaptive boosting), SVM, and K$\mathrm{NN}$ classifiers to determine the device efficiency of the system. According to the article [13 14], a deep learning neural network was used to examine the EEG data. Using $\mathrm{CNN}$, the features were extracted and they were assigned to each of the three types of seizure classifications, before, during, and after the seizure.

In the detection of real-time seizure activity from intracranial EEG signals was applied with a new method called machine learning, which used the prior seizure observations to arrive at an optimal or expected seizure detection threshold. We had to get 
spectra and time stamps for each photo and extract temporal and spectral features to train the pattern recognition. Utilizing linear discriminant analysis and wavelet wavelet transform-based features, [15] as well as triadic wavelet transform discriminants, K-NN classifiers were used to identify the seizure signals the main goal of this study is to discover if seizures are present due to epilepsy. These systems provide means of intercession for those who were never affected by the seizure to be monitored as well as systems that surgery or treatment has zero effects. Because of their architectures, above-constructed systems also require feature extraction and classification steps, the word we're looking for can be found using a method known as a multi-layered query The methods used in this research, for seizure identification on the scalp, were used in $\mathrm{CNN}$ as well to locate the seizure originating in the body [16] [17]. CNN works with unsupervised learning that automatically looks for representation layers within a collection of feature databases [that have been created by previous layers of processing] and learning features naturally within the $\mathrm{CNN}$, as it learns to identify them an empirical study of artificial intelligence, known as the deep learning, has confirmed its ability and shown itself to outclass human cognition on the difficult problems of image and audio recognition $[18,19]$. A related to machine learning has been applied in a number of diverse ways, such as diagnosing Alzheimer earlier, calculating the degree of illness, and determining the severity of diseases in the chest, all of Algorithms, and strength assessment.

According to MansiAgrawal et al. [21] proposed a system of hand gesture identification using deep $\mathrm{CNN}$, this module they proposed a collect the streaming data from a live webcam and converted it into various frames. Then using preprocessing and gesture sign recognition, the final signal has been indeed using soft computing techniques. According to Swati Avad and DrSharmishta Desai [22] proposed a system of Indian sign language recognition using machine learning techniques; this system is the collaboration of image processing techniques and machine learning approaches. The initial image processing techniques, life segmentation, histogram generation, feature extraction and selection and finally, classification process has done for final detection.

According to Meet Patel, Sharmishta Desai [23] proposed a system music recommendation for paralyzed peoples; this system author has collected a brain EEG signal in the runtime environment and classify the emotional using deep learning-based CNN algorithms. This system has evaluated synthetic and teal time signals data that produces high accuracy over the traditional machine learning algorithms.

According to DrSharmishta Desai [24], proposed student behaviour analysis using dynamic induction module, The decision tree algorithm visualizes data, which is beneficial for social media data analysis. Since they store all data in memory for constructing a model, neural networks like C4.5 or CART have a memory designed to regulate. As a result, these algorithms are ineffective when dealing with large amounts of data. When the amount of data is small, these algorithms perform well, but the algorithms perform poorly as the amount of data grows. We used the Hoeffding tree for a large volume of data in this paper, and the results show that the Hoeffding tree outperforms other Data Mining algorithms.

DrSharmishta Desai [25] proposed a framework for analyzing consumer data from social media sites that compared the performance of various supervised learning models to market data. The Clustering method with one-level logistic regression is found to work the best against trade data. They used AdaBoost to enhance the productivity of decision trees, and the results were impressive. The various stages of large-scale data extraction are also thoroughly clarified. 


\section{Proposed works}

In proposed research work evaluate the entire system with supervised learning algorithms, initially collect the data from the brain as EEG signals. To extract the various features from inputs Set and generate the trained model accordingly from CNN and LSTM algorithm. The objective of the system to identify the epileptic disease based on given ECG signals. In the testing system classify each input signal with respective labels and show the system efficiency.

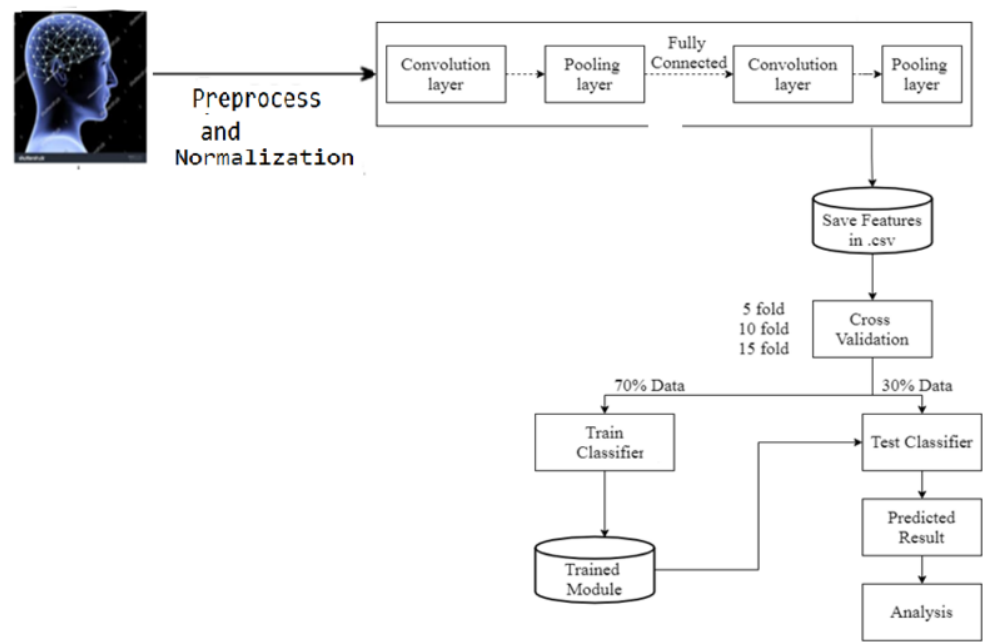

Figure 1. Proposed system overview

Features from the training data have been examined and training models have been created, which has the various specific capabilities as a result. Another image feature extraction technique is implemented on the testing dataset, which extracts each feature only if it'svalue exceeds a certain threshold. The feature weight identification method involves determining the similarities between testing and training conditions. When comparing two features sets, we go deeper into the data in order to discover what they have in common. The weight factor takes values between which corresponds to a person's preference for objects and attaches them to those values. Initial weight is 0 , while the threshold can be system defined.

CNN-LSTM classifier has used for classification of epileptic disease in proposed architecture. It is a supervised learning model, used mainly for study on classification and regression. The classification frequently used for challenges regarding object recognition, performs particularly well in aspect-based applications recognition, and classification based on colour. Encourage vector computers aren't better than others machine learning approaches; however, they do at the state-of-the-art, and with a lot of current both conceptual and empirical. CNN-LSTM is known as by many researchers as a better algorithm for performing rankings. The EEG Input signals the values are entered in the classifier and the epileptic disease are classified by hyper plane using drawn with the epileptic disease identified. 


\section{Observations}

- From the above literature review, several systems deal with deep convolutional neural networks, and various boosting approaches have been used to improve computational complexity.

- To achieve a better time and space complexity, a convolutional neural network and various types of boosting methods were used.

- To classify the sentiment of stratified image datasets, researchers used region-based convolutional neural networks and other computer program algorithms such as PNN, RNN, and DCNN.

- The features were extracted using the ImageNet library, and the transport model was built accordingly. It can be difficult to achieve better precision than $\mathrm{CNN}$ using PNN, RNN, and other techniques.

- It is proposed that a combination of fast recurrent neural networks and CNN yield the highest accuracy with the least amount of time complexity.

- When a multi convolution layers is formed, it takes a long time for $\mathrm{CNN}$ to produce, and it also takes a lot of data when the system would be dealing with heterogeneous datasets. Irrelevant features are removed by function extraction techniques, resulting in high dimensionality problems.

- Several current studies on CNN have found that the average accuracy for text categorization on the flicker picture dataset is about 96.50 percent.

\section{Results and Discussions}

Getting equipped to efficiently perceive emotions would be Important for decision support structures such as Mental Health conditions which is a method for controlling risk and protection used by Practitioners in mental health and people for evaluation. Emotional evaluations, independent of self-reporting would help calibrate and guide the advice further delicate encounters. Brainwave analysis applied it is too invasive in this paper but would be useful to have a benchmark for discovering other approaches which are more acceptable. The implementation has done in python base open-source platform. Below table 1 shows the classification accuracy with two different algorithms respectively.

Table 1. Accuracy of system using KNN, SVM and CNN

\begin{tabular}{|c|c|c|}
\hline Algorithm & Accuracy & Dataset \\
\hline \multirow{2}{*}{ KNN } & $56.25 \%$ & Synthetic \\
\cline { 2 - 3 } & $56.64 \%$ & EEG-IoT \\
\hline \multirow{2}{*}{ SVM } & $65.25 \%$ & Synthetic \\
\cline { 2 - 3 } & $64.45 \%$ & EEG-IoT \\
\hline \multirow{2}{*}{ CNN } & $92.50 \%$ & Synthetic \\
\cline { 2 - 3 } & $93.60 \%$ & EEG-IoT \\
\hline
\end{tabular}




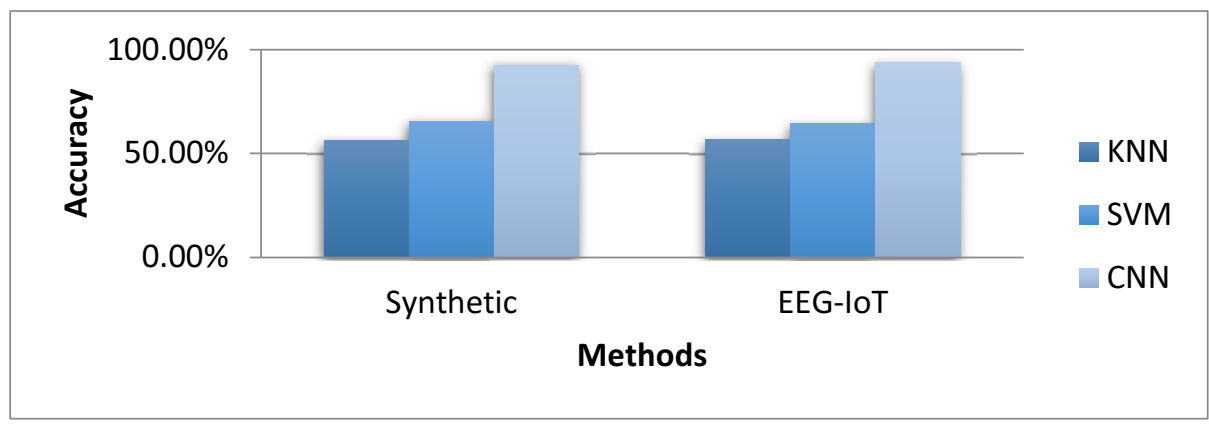

Figure 2. Accuracy of proposed system using KNN and SVM algorithm

The proposed system used KNN, SVM and CNN three different machine learning and deep learning classifiers which are evaluated with different cross-validation of realtime EEG datasets. Various feature extraction techniques have been carried out during the model training and a similar strategy has been applied for testing. The above Figure 2 demonstrates synthetic and real time IoT dataset.

\section{Conclusion}

Our research looks at the use of deep learning techniques in BCI Emotions classification systems. Images can be used in a variety of ways, including programmed marking with sentimental parts in order, automatically classifying video scenes into thrillers, comedy, romance, and other genres, and automatically classifying video classes with emotions categories. According to the results of the survey, CNN-LSTM can be created with different confusion matrix parameters when dealing with different datasets. With more than 96 percent average classification accuracy, DCNN using the RESNET library achieves the highest accuracy.

Images from one specific event have been effective with allusions to particular news organizations such as $\mathrm{CNN}$ has already been demonstrated in a lot of experiments, particularly the context of metaphor construction. Since the CNN-LSTM architectures were used to classify the EEG data, we take these into consideration as well. In order to use the unprocessed input data as training and development data, we first expanded it into a two-dimensional representation. The researchers developed an effective and cutting-edge method of using only visual, auditory, and textual features to accurately and easily identify human emotions. The best features of the Full Feature Equivalent from a long feature table are chosen and used to expand the network to achieve high-quality results faster expansion. Here, we use wavelet-based transforms to translate one-dimensional signals into two-transformed time-frequency representations. Features from the four models are combined to reduce the function vector's scale by including negative information in the proposal calls for the reduction of the number of channels via differential entropy. A model will develop that is several steps ahead of previous models in its ability to obtain functionality. The classifier's efficiency has improved greatly over the preceding versions. Expansion also makes it possible for feature detection and real-time recognition of emotions by using an EEG. According to this work, it appears that neural networks and deep learning can be combined in order to develop a complex model. Further study is still remaining to be done to investigate the emotional analysis, to be performed by the combined use of many neural networks. 


\section{References}

[1]. Ghosh-Dastidar, S.; Adeli, H.; Dadmehr, N. Mixed-band wavelet-chaos-neural network methodology for epilepsy and epileptic seizure detection. IEEE Trans. Biomed. Eng. 2007, 54, 1545-1551.

[2]. Polat, K.; Güne, s, S. Classification of epileptiform EEG using a hybrid system based on decision tree classifier and fast Fourier transform. Appl. Math. Comput. 2007, 187, 1017-1026.

[3]. Liang, S.F.; Wang, H.C.; Chang, W.L. Combination of EEG complexity and spectral analysis for epilepsy diagnosis and seizure detection. EURASIP J. Adv. Signal Process.2010, 1, 853434.

[4]. Faust, O.; Acharya, U.R.; Adeli, H.; Adeli, A. Wavelet-based EEG processing for computer aided seizure detection and epilepsy diagnosis. Seizure 2015, 26, 56-64.

[5]. Acharya, U.R.; Sree, S.V.; Chattopadhyay, S.; Yu, W.; Ang, P.C.A. Application of recurrence quantification analysis for the automated identification of epileptic EEG signals. Int. J. Neural Syst. 2011, 21, 199-211.

[6]. Bajaj, V.; Pachori, R.B. Classification of seizure and nonseizure EEG signals using empirical mode decomposition. IEEE Trans. Inf. Technol. Biomed. 2012, 16, 1135-1142.

[7]. Andrzejak, R.G.; Lehnertz, K.; Rieke, C.; Mormann, F.; David, P.; Elger, C.E. Indications of Nonlinear Deterministic and Finite Dimensional Structures in Time Series of Brain Electrical Activity: Dependence on Recording Region and Brain State. Phys. Rev. E 2001, 64, 061907.

[8]. Adeli, H.; Ghosh-Dastidar, S.; Dadmehr, N. A wavelet-chaos methodology for analysis of EEGs and EEG sub-bands to detect seizure and epilepsy. IEEE Trans. Biomed. Eng. 2007, 54, 205-211.

[9]. Kabir, E.S.; Zhang, Y. Epileptic seizure detection from EEG signals using logistic model trees. Brain Inform. 2016, 3, 93-100.

[10]. Subasi, A.; Gursoy, M.I. EEG signal classification using PCA, ICA, LDA and support vector machines. Expert Syst. Appl. 2010, 37, 8659-8666.

[11]. Richhariya, B.; Tanveer, M. EEG signal classification using Universum support vector machine. Expert Syst. Appl. 2018, 106, 169-182.

[12]. Raghu, S.; Sriraam, N. Classification of focal and non-focal EEG signals using neighbourhood component analysis and machine learning algorithms. Expert Syst. Appl. 2018, 15, 18-32.

[13]. Acharya, U.R.; Oh, S.L.; Hagiwara, Y.; Tan, J.H.; Adeli, H. Deep convolutional neural network for the automated detection and diagnosis of a seizure using EEG signals. Comput. Biol. Med. 2018, 100, 270 278.

[14]. Truong, N.D.; Nguyen, A.D.; Kuhlmann, L.; Bonyadi, M.R.; Ippolito, J.Y.S.; Kavehei, O. Convolutional neural networks for seizure prediction using intracranial and scalp electroencephalogram. Neural Netw. 2018, 105, 104-111.

[15]. Shoeb, A.H. Application of Machine Learning to Epileptic Seizure onset Detection and Treatment. Ph.D. Thesis, Massachusetts Institute Technology, Massachusetts, UK, 2009.

[16]. Kharbouch, A.; Shoeb, A.; Guttag, J.; Cash, S.S. An algorithm for seizure onset detection using intracranial EEG.Epilepsy Behav. 2011, 22, 529-535.

[17]. Chandel, G.; Upadhyaya, P.; Farooq, O.; Khan, Y.U. Detection of Seizure Event and Its Onset/Offset Using Orthonormal TriadicWavelet Based Features. IRBM 2019, 40, 103-112.

[18]. LeCun, Y.; Bengio, Y.; Hinton, G. Deep Learning. Nature 2015, 521, 436-444.

[19]. Krizhevsky, A.; Sutskever, I.; Hinton, G.E. ImageNet classification with deep convolutional neural networks. ACM Commun. 2017, 60, 84-90.

[20]. Ortiz-Garcia, A.; Munilla, J.; Gorriz, J.M.; Ramirez J. Ensembles of deep learning architectures for the early diagnosis of Alzheimer's disease. Int. J. Neural Syst. 2016, 26, 1650025.

[21]. ManasiAgrawal, RutujaAinapure, ShrushtiAgrawal, SimranBhosale, Sharmishta Desai "Models for Hand Gesture Recognition using Deep Learning”, 2020 IEEE 5th International Conference on Computing Communication and Automation (ICCCA),589-594.

[22]. Swati Avad, Dr. Sharmishta Desai "A research review on Indian Sign Language Recognition Using Machine Learning", Journal of Computer Science Engineering and Software Testing (e-ISSN: 25816969) Volume 6 Issue 2 Year 2020.

[23]. Meet Patel, Sharmishta Desai "Music suggestion according to emotion for paralyzed and bed rest patient using EEG Signals", Journal of SeyBold report, July 2020,1533-9211.

[24]. Dr.Sharmishta Desai “Dynamic Induction Model for Student's Behavior Analysis",International Journal of System and Software Engineering,7(1), June 2019, 13-19.

[25]. Sharmishta Desai, Dr.S.T.Patil "Boosting Decision Trees for Prediction of Market Trends,Journal of Engineering and Applied Sciences”,552-556,April 2018,ISSN:1818-7803. 